\title{
News Shocks, Information Flows and SVARs
}

\section{PATRICK FEVE AND AHMAT JIDOUD}




\title{
News Shocks, Information Flows and SVARs
}

\author{
Patrick Feve* \\ Toulouse School of Economics (GREMAQ, IDEI, IUF and Banque de France) \\ Ahmat Jidoud \\ Toulouse School of Economics (GREMAQ)
}

March, 2012

\begin{abstract}
This paper assesses SVARs as relevant tools at identifying the aggregate effects of news shocks. When the econometrician and private agents' information sets are not aligned, the dynamic responses identified from SVARs are biased. However, the bias vanishes when news shocks account for the bulk of fluctuations in the economy. A simple correlation diagnostic test shows that under this condition, news shocks identified through long-run and short-run restrictions have a correlation close to unity.
\end{abstract}

Keywords: News shocks, Information Flows, Non-fundamentalness, SVARs, Identification

JEL Class.: C32, C52, E32

\section{Introduction}

Given the increasing interest towards the empirical relevance of news shocks (see e.g. Beaudry and Portier, 2005, 2006 and Beaudry and Lucke, 2009) and the widespread use of structural vector autoregresive (SVAR) models as relevant tools for the the identification of economic shocks, we assess the ability of SVARs at correctly uncovering news shocks. Identifying news shocks in SVARs happens to be a tedious task because foresight (news) creates an equilibrium (and thus data) in which the econometrician and agents' information sets are not aligned (see Feve, Matheron and Sahuc, 2009, and Leeper, Walker and Yang, 2011). Such information misalignment alters the identification of structural shocks from past and current data, an assumption taken as given in VAR analysis.

\footnotetext{
*Address: Université de Toulouse 1, Aile Jean-Jacques Laffont, 21 Allée de Brienne, 31000, Toulouse, France. email: patrick.feve@univ-tlse1.fr. We would like to thank M. Dupaigne, R. Farmer, J-P. Florens, J. Matheron, M. Normandin, R. Pancrazi, P. Pintus, F. Portier, G. Saint-Paul, J-G. Sahuc and A. Seymen for helpful comments and suggestions. The views expressed herein are those of the authors and should not be interpreted as reflecting those of the Banque de France.
} 
We follow the identification strategy adopted by Beaudry and Portier (2006) and investigate under which conditions SVARs may properly uncover the true dynamic responses to news shocks. We estimate a VAR model with two observed variables under a Data Generating Process (DGP) represented by a simple dynamic model with rational expectations. In spite of its abusive simplicity, the structural model provides insightful guidance about the identification of news shocks in a SVAR framework. Following Beaudry and Portier (2006), we impose sequentially long-run and short-run restrictions in the VAR model and then compute a simple correlation diagnostic test.

We find that the accuracy of the dynamics implied by SVARs solely depends on the relative contribution of news shocks in driving fluctuations. As long as this contribution is large, these dynamics are consistently estimated and the identified structural shocks (using long-run and short-run restrictions) display a strong positive correlation.

The paper is organized as follows. In a first section, we expound our reference setup and discuss non-fundamentalness issues. In a second section, we report the identified dynamic responses using both long-run and short-run restrictions. A last section concludes.

\section{A Simple Model with Expected Shocks}

\subsection{The Model}

The model economy takes the following form

$$
\begin{aligned}
y_{t} & =a E_{t} y_{t+1}+b E_{t} \Delta x_{t+1}+w_{t}, \quad|a|<1, \quad b \neq 0 \\
\Delta x_{t} & =\sigma_{\varepsilon} \varepsilon_{t-q}, \\
w_{t} & =\sigma_{u} u_{t},
\end{aligned}
$$

where $\sigma_{\varepsilon}>0$ and $\sigma_{u}>0 . y_{t}$ is a single endogenous variable. $E_{t}$ denotes the conditional expectation operator. Two shocks hit the economy. The first shock $x_{t}$ is specified in first difference and represents the non-stationary component of the economy. The second shock $w_{t}$ is stationary. Their innovations $\varepsilon_{t}$ and $u_{t}$ in (2) and (3) are serially uncorrelated with zero mean and unit variance. They are mutually uncorrelated for all leads and lags. Equation (1) naturally emerges from optimization problem in stochastic equilibrium models. Equation (2) is the backbone of our analysis. It can be associated with Total Factors Productivity (TFP) growth, exogenous dividend growth or any forcing variables, depending on the structural model considered. When taking their decisions in period $t$, agents in the economy have perfect expectations about $x_{t}$ in the next $q$ periods. Thus the information set of the agents is given by all the history of $\Delta x_{t}$ and its foreseen values for the next $q$ periods: $I^{A}=\left(\varepsilon_{t}, \varepsilon_{t-1}, \ldots\right)$. However using the VAR model with the observations on $\left\{\Delta x_{t}, y_{t}\right\}$ restricts the 
econometrician's information set to be $I^{E}=\left(\varepsilon_{t-q}, \varepsilon_{t-q-1}, \ldots\right) \subset I^{A}$.

Excluding sunspots (i.e. we impose $|a|<1$ ) and bubbles (i.e. we restrict the solution to satisfy $\left.\lim _{T \rightarrow \infty} E_{t} a^{T} y_{t+T}=0\right)$ and using the processes (2) and (3), we obtain the solution for $y_{t}$

$$
y_{t}=b \sigma_{\varepsilon} \sum_{i=0}^{q-1} a^{q-1-i} \varepsilon_{t-i}+\sigma_{u} u_{t}
$$

Equation (4) together with equation (2) represent the DGP. We assume that the variables $\Delta x_{t}$ and $y_{t}$ are observed by the econometrician, while the variable $w_{t}$ is not. ${ }^{1}$ Equations (2) and (4) rewrite

$$
Z_{t}=\mathcal{H}(L) v_{t}
$$

where $Z_{t}=\left(\Delta x_{t}, y_{t}\right)^{\prime}$ and $v_{t}=\left(\varepsilon_{t}, u_{t}\right)^{\prime}$. The matrix $\mathcal{H}(L)$ is given by

$$
\mathcal{H}(L)=\left(\begin{array}{cc}
\sigma_{\varepsilon} L^{q} & 0 \\
b \sigma_{\varepsilon} \sum_{i=0}^{q-1} a^{q-1-i} L^{i} & \sigma_{u}
\end{array}\right),
$$

where $L$ is a backshift operator.

\subsection{Structural VAR and Non-fundamentalness}

One of the principal arguments motivating the question at hand is that VAR models face troublesome concerns when they are used in an environment in which private agents receive new information today about future developments in the economy. Such a situation results in non-fundamental representations of the VAR model because by employing a VAR representation, the econometrician has unknowingly conditioned on a smaller information set (see Leeper et al, 2011). ${ }^{2}$ It is easy to check that system (5) is non-fundamental. Indeed, the determinant of its characteristic polynomial satisfies $\operatorname{det} \mathcal{H}(z)=\sigma_{\varepsilon} \sigma_{u} z^{q}$ whose unique root is zero when $q \neq 0$. Consequently the system (5) is nonfundamental so long as $q \geq 1$. Furthermore, the non-fundamentalness is driven by the misalignment of information sets and it is not directly related to the moving-average component in the process of $y_{t}$. In this sense, our results line up with those of Leeper et al (2011). Indeed, if instead of $Z_{t}=\left(\Delta x_{t}, y_{t}\right)^{\prime}$, one considers $Z_{t}^{q}=\left(\Delta x_{t+q}, y_{t}\right)^{\prime}$, then the resulting system is fundamental because the determinant of the corresponding matrix is equal to $\sigma_{\varepsilon} \sigma_{u}$. This shows that accounting explicitly for the information flows in the specification of the VAR model solves the non-invertibility issue. ${ }^{3}$ For sake of tractability of the computations, we consider a one period news shock $(q=1)$ as this is enough to generate a non-fundamental VAR representation. In this case, the DGP for $\left\{\Delta x_{t}, y_{t}\right\}$ is given by $\Delta x_{t}=\sigma_{\varepsilon} \varepsilon_{t-1}$ and $y_{t}=b \sigma_{\varepsilon} \varepsilon_{t}+\sigma_{u} u_{t}$.

\footnotetext{
${ }^{1}$ We closely follow the empirical strategy adopted by Beaudry and Portier (2005) and (2006) by assuming that the variable subject to news shocks is observed, together with $y_{t}$.

${ }^{2}$ Non-fundamentalness issues have been already deeply discussed in rational expectation econometrics (see the references in Leeper, Walker and Yang, 2011)

${ }^{3}$ This finding suggests to use $Z_{t}^{q}$ instead of $Z_{t}$ in the VAR model. In this case, the SVAR model perfectly uncovers the true shock in our setup.
} 


\section{Estimation and Identification}

\subsection{Estimation}

All of our results are obtained from the probability limit of the moments associated to $\Delta x_{t}$ and $y_{t}$. We consider a $\operatorname{VAR}(p)$ model as a simple way to statistically represent the solution of our structural model, where $p$ is the number of lags. Linear projections of $\Delta x_{t}$ and $y_{t}$ on their lagged values implies a value of $p=1.4$ This means that any bias in the estimated responses is not the consequence of a truncated dynamics due to an insufficient number of lags in the VAR model. The estimated model writes as follows:

$$
Z_{t}=A Z_{t-1}+v_{t}
$$

where $v_{t}=\left(v_{1, t}, v_{2, t}\right)^{\prime}$ is the vector of VAR disturbances. Using moments generated by our DGP, the matrix $A$ and the covariance matrix $\Omega_{v}$ of the canonical innovations are given by

$$
A=\left(\begin{array}{cc}
0 & \frac{\omega}{b} \\
0 & 0
\end{array}\right) \quad, \quad \Omega_{v}=\left(\begin{array}{cc}
\frac{\omega \sigma_{u}^{2}}{b^{2}} & 0 \\
0 & b^{2} \sigma_{\varepsilon}^{2}+\sigma_{u}^{2}
\end{array}\right)
$$

where $\omega=\left(b^{2} \sigma_{\varepsilon}^{2}\right) /\left(b^{2} \sigma_{\varepsilon}^{2}+\sigma_{u}^{2}\right) \equiv V(y \mid \varepsilon) / V(y)$ measures the relative contribution of news shocks to the total variance $y_{t}$ in the DGP. $\omega$ lies in $(0,1)$ and decreases with the relative size of standard surprise shocks with respect to news shocks. Its central role in this paper will become shortly obvious. ${ }^{5}$

\subsection{Identification}

Following the empirical strategies developed by Beaudry and Portier (2006) for the identification of news shocks, we consider sequentially long-run and short-run restrictions.

Long-Run Restriction Let $B(L)=\left(I_{2}-A L\right)^{-1}$ and $\eta_{t}=\left(\eta_{1, t}, \eta_{2, t}\right)^{\prime}$ be the vector of structural shocks. As usual, we impose an orthogonality assumption on the structural shocks, which combined with a scale normalization implies $\operatorname{Var}\left(\eta_{t}\right)=I_{2}$. We thus have the structural VMA representation $Z_{t}=$ $B(L) v_{t}=C(L) \eta_{t}$, where $C(L)=B(L) S$ and $S$ is a non-singular matrix constructing the innovations $v_{t}$ as linear combinations of structural disturbances $\eta_{t}$. Following Blanchard and Quah (1989), we impose a long-run restriction on the matrix $C(1)=B(1) S$. The orthogonality and normalization assumptions on the two structural shocks through $\operatorname{Var}\left(\eta_{t}\right)=I_{2}$ provide a first set of restrictions to uncover some parameters. However, this is not enough to fully recover the effects of all shocks and thus an additional exclusion restriction is imposed upon the long-run response of $x_{t}$. Given the

\footnotetext{
${ }^{4} \mathrm{~A}$ full characterization of all the results can be obtained from the authors.

${ }^{5}$ Notice that the estimated VAR model under our DGP implies that the endogenous variable $y_{t}$ Granger causes the exogenous variable $\Delta x_{t}$. This represents an additional illustration of pitfalls in using causality tests.
} 
ordering of $Z_{t}$, we simply require that $C(1)$ be lower triangular, so that only news shocks can affect the long-run level of $x_{t}$. This amounts to imposing that $C(1)$ be the Cholesky factor decomposition of the long-run covariance matrix $\Sigma^{L R}=C(1) C(1)^{\prime}$, where $\Sigma^{L R}=B(1) \Omega_{v} B(1)^{\prime}$. Given this identity, we can easily recover $C(1)$ and accordingly $S=B(1)^{-1} C(1) \equiv(I-A) C(1)$. Furthermore we have $C(L)=B(L) S=B(L) B(1)^{-1} C(1)$. The SVAR model admits the following representation

$$
\left(\begin{array}{c}
\Delta x_{t} \\
y_{t}
\end{array}\right)=\left(\begin{array}{cc}
\sigma_{\varepsilon}(1-\omega) & -\omega \frac{\sigma_{u}}{b} \\
b \sigma_{\varepsilon} & \sigma_{u}
\end{array}\right)\left(\begin{array}{c}
\eta_{1, t} \\
\eta_{2, t}
\end{array}\right)+\left(\begin{array}{cc}
\sigma_{\varepsilon} \omega & \omega \frac{\sigma_{u}}{b} \\
0 & 0
\end{array}\right)\left(\begin{array}{l}
\eta_{1, t-1} \\
\eta_{2, t-1}
\end{array}\right) .
$$

System (7) allows us to compute the dynamic responses to the identified news shock. These are are given by

$$
\begin{gathered}
\frac{\partial \Delta x_{t}}{\partial \eta_{1 t}}=(1-\omega) \sigma_{\varepsilon} \geq 0 \quad, \quad \frac{\partial \Delta x_{t+1}}{\partial \eta_{1 t}}=\omega \sigma_{\varepsilon} \leq \sigma_{\varepsilon} \quad \text { and } \quad \frac{\partial \Delta x_{t+h}}{\partial \eta_{1 t}}=0 \quad \forall h \geq 2, \\
\frac{\partial y_{t}}{\partial \eta_{1 t}}=b \sigma_{\varepsilon} \text { and } \frac{\partial y_{t+h}}{\partial \eta_{1 t}}=0 \quad \forall h \geq 1 .
\end{gathered}
$$

It turns out that the SVAR model fails to perfectly mimic the dynamics of the model economy especially those of the exogenous variable $x_{t} .{ }^{6}$ Though the DGP postulates the presence of news in the economy, the SVAR model is unable to detect them as it overestimates the impact response of $x_{t}$ to the news shock. Like the impact response, the one period response is biased. The size of these biases hinges however on the relative contribution of news shocks in driving aggregate fluctuations, i.e on $\omega$. The larger this contribution (i.e $\sigma_{\varepsilon}>>\sigma_{u}$ ) the closer is $\omega$ to unity and the smaller are the biases. Only in this case, could the SVAR be appropriately used as a relevant tool to identify news shocks. Interestingly, however, the econometrician perfectly uncovers the true dynamics of the endogenous variable $y_{t}$ independently on whether news shocks are the dominant source of its fluctuations.

Short-Run restrictions In this setup, the econometrician uses a prior information and imposes a restriction on the impact response of $\Delta x_{t}$ (Sims, 1980). The restriction concerns now the matrix $\tilde{C}(0)=B(0) \tilde{S} \equiv \tilde{S}$ which gives the impact of shocks in the short-run. Notice that we impose again an orthogonality assumption on the structural shocks and a scale normalization, as in the previous SVAR setup. News shocks are assumed to have a zero impact on $\Delta x_{t}$. This corresponds to $\tilde{s}_{11}=0$ (the $(1,1)$ entry of the matrix $\tilde{S})$. The previous system rewrites as $Z_{t}=B(L) v_{t}=\tilde{C}(L) \tilde{\eta}_{t}$, where the vector of innovations is now $\tilde{\eta}_{t}=\left(\tilde{\eta}_{1, t}, \tilde{\eta}_{2, t}\right)^{\prime}$. The matrix $\tilde{S}$ verifies $\tilde{S} \tilde{S}^{\prime}=\Omega_{v}$, i.e. $\tilde{S}$ is a Cholesky decomposition of the variance covariance matrix $\Omega_{v}$ of the canonical residuals. Using this decomposition, we obtain

$$
\left(\begin{array}{c}
\Delta x_{t} \\
y_{t}
\end{array}\right)=\left(\begin{array}{cc}
0 & \frac{\sigma_{u}}{b} \sqrt{\omega} \\
\frac{b \sigma_{\varepsilon}}{\sqrt{\omega}} & 0
\end{array}\right)\left(\begin{array}{l}
\tilde{\eta}_{1 t} \\
\tilde{\eta}_{2 t}
\end{array}\right)+\left(\begin{array}{cc}
\sigma_{\varepsilon} \sqrt{\omega} & 0 \\
0 & 0
\end{array}\right)\left(\begin{array}{l}
\tilde{\eta}_{1, t-1} \\
\tilde{\eta}_{2, t-1}
\end{array}\right) .
$$

\footnotetext{
${ }^{6}$ Remember that the true dynamics responses of $\Delta x_{t}$ to a news shocks are $\partial \Delta x_{t} / \partial \varepsilon_{t}=0, \partial \Delta x_{t+1} / \partial \varepsilon_{t}=\sigma_{\varepsilon}$ and $\partial \Delta x_{t+h} / \partial \varepsilon_{t}=0, \forall h \geq 2$ (the cumulative responses are $\partial x_{t} / \partial \varepsilon_{t}=0$ and $\partial x_{t+h} / \partial \varepsilon_{t}=\sigma_{\varepsilon}, \forall h \geq 1$ ). In addition, the true dynamics responses of $y_{t}$ to a news shocks are $\partial y_{t} / \partial \varepsilon_{t}=b \sigma_{\varepsilon}$ and $\partial \Delta y_{t+h} / \partial \varepsilon_{t}=0, \forall h \geq 1$.
} 
The estimated dynamic responses are deduced from the system (8)

$$
\begin{gathered}
\frac{\partial \Delta x_{t}}{\partial \tilde{\eta}_{1 t}}=0, \quad \frac{\partial \Delta x_{t+1}}{\partial \tilde{\eta}_{1 t}}=\sigma_{\varepsilon} \sqrt{\omega} \leq \sigma_{\varepsilon} \quad \text { and } \quad \frac{\partial \Delta x_{t+h}}{\partial \tilde{\eta}_{1 t}}=0 \quad \forall h \geq 2, \\
\frac{\partial y_{t}}{\partial \tilde{\eta}_{1, t}}=\frac{b \sigma_{\varepsilon}}{\sqrt{\omega}} \geq b \sigma_{\varepsilon} \text { and } \frac{\partial y_{t+h}}{\partial \tilde{\eta}_{1 t}}=0 \quad \forall h \geq 1 .
\end{gathered}
$$

As a direct consequence of the identification scheme, the impact response of $\Delta x$ is zero. This identification strategy is indeed consistent with the hypothesis that the econometrician truly knows the timing of news. Besides, the remaining relevant dynamics are biased. Once again, the size of the discrepancy between the estimated and the structural dynamics is governed by the relative importance of news shocks. As $\omega \rightarrow 1$, an econometrician using a SVAR with short-run restrictions bears little risk of missing the structural dynamics.

\subsection{The Correlation Diagnostic Test}

Using actual US data, Beaudry and Portier (2006) have performed a test allowing to assess the empirical plausibility of the news shock hypothesis. This simple diagnostic test consists in computing the correlation between the identified news shocks recovered from long-run and short-run restrictions ( $\eta_{1, t}$ and $\tilde{\eta}_{1, t}$ in our previous notations) and see how this correlation evolves. ${ }^{7}$ We adopt this strategy and evaluate its relevance in our set-up. With regard to the previous SVARs, the identified shocks $\eta_{1, t}$ and $\tilde{\eta}_{1, t}$ can be expressed as functions of the two structural shocks $\varepsilon_{t}$ and $u_{t}$ of the DGP. Hence, $\eta_{1, t}=(1-\omega) \varepsilon_{t-1}+\omega \varepsilon_{t}-\left(b \sigma_{\varepsilon} / \sigma_{u}\right)(1-\omega)\left(u_{t}-u_{t-1}\right)$ and $\tilde{\eta}_{1, t}=\sqrt{\omega} \varepsilon_{t}+\left(\sigma_{u} /\left(b \sigma_{\varepsilon}\right)\right) u_{t}$. Direct calculations yield

$$
\operatorname{corr}\left(\eta_{1 t}, \tilde{\eta}_{1 t}\right)=\sqrt{\omega}
$$

This correlation is positive and is an increasing function of the key parameter $\omega$, i.e. the contribution of news shocks to the variance of $y_{t}$. As long as $\sigma_{\varepsilon} \gg \sigma_{u}$, the correlation tends to unity. This result shows that the two identification strategies provide accurate estimates of the dynamic responses and a relevant diagnostic test for the identification of news shocks, both of them evolving in the same direction with the key parameter $\omega$.

\section{Concluding Remarks}

In this paper, we assesses the ability of SVARs at identifying the aggregate effects of news shocks. We show that, although there are biases in the estimated responses to news shocks, these biases are no longer significant when news shocks account for a substantial part of fluctuations in the economy.

\footnotetext{
${ }^{7}$ Beaudry and Portier (2006) obtain a correlation close to one and conclude that this result strongly supports their empirical findings about the relevance of news shocks.
} 
Moreover, we find that the correlation between the identified innovations is almost equal to one when expected shocks are the dominant source of fluctuations.

\section{References}

Beaudry, P. and F. Portier, (2005) "The news view of ecconomic fluctuations: evidence from aggregate Japanese data and sectoral US data", Journal of the Japanese and International Economics, 191, 635652.

Beaudry, P. and F. Portier, (2006) "Stock prices, news and economic fluctuations", American Economic Review, 96, 96(4): pp. 1293-1307.

Beaudry, P. and B. Lucke, (2009) "Letting different views about business fluctuations compete", NBER Macroeconomics Annual 2009, Vol. 24, pp. 413-455

Blanchard, O.J. and D. Quah (1989) "The Dynamic Effects of Aggregate Demand and Supply Disturbances", American Economic Review, 79(4), pp. 655-673.

Fève, P., J. Matheron, and J-G. Sahuc, (2009) "On the Dynamic Implications of News Shocks", Economic Letters, 102, pp. 96-98

Leeper, E., T. Walker, and S. Yang, (2011) "Foresight and Information Flows", NBER Working Paper 16951.

Sims, C. (1980) "Macroeconomics and Reality", Econometrica, 48, pp. 1-48. 